Short communication

\title{
A note on the clonal propagation of depleted threatened species Boswellia serrata Roxb. through branch cuttings
}

\author{
Vivek Vaishnav ${ }^{1} *$ and Ugendra Janghel ${ }^{2}$ \\ ${ }^{1}$ Institute of Forest Productivity, Ranchi-835303, Jharkhand, India \\ ${ }^{2}$ State Forest Research and Training Institute, Raipur-493111, Chhattisgarh, India \\ *Corresponding Author: vivekvaishnaw@ live.in \\ [Accepted: 27 March 2018]
}

[Cite as: Vaishnav V \& Janghel U (2018) A note on the clonal propagation of depleted threatened species Boswellia serrata Roxb. through branch cuttings. Tropical Plant Research 5(1): 27-28]

\section{INTRODUCTION}

Boswellia serrata Roxb. (Family- Burseraceae) commonly known as salai guggul or Indian olibanum, is an important deciduous medicinal tree endemic to India (Kapoor 1990, Shah et al. 2008) (Fig. 1). The oleo-gumresin obtained by an incision made on the trunk of the tree contains boswellic acid which is effective for the treatment of an inflammatory disorder such as arthritis and in cardiovascular diseases (Ammon 2006). Apart from these, due to long wood fiber, the pulpwood was extremely utilized for paper mills like Nepanagar paper mill of Madhya Pradesh (Khan 1972).

The species is self-incompatible and the self-crossed pollen tubes are inhibited soon after their entry into the stigma. Cross-pollinated flowers allowed normal pollen germination and pollen tube growth, and resulted in fruit- and seed-set (Sunnichan et al. 2005). But poor fruit setting (2.6 to 10\%) under open pollination, inadequate production of viable seeds (Joshi 1981) and scanty seed germination (10-20\%) restrict the distribution and therefore limits its natural source (Purohit et al. 1995, Ghorpade et al. 2010). On the other hand, the tree is harvested to extract the sap and to utilize the pulp in paper industries. Such problematic regeneration and the destructions have resulted in declining of the natural abundance and the species has been classified under threatened (Saha et al. 2015). Therefore, development of mass multiplication technique for conservation and sustainable utilization of this species has become a serious concern. The present experiment was performed to standardize the propagation technique of the species transferable to nurseries of forest departments.

\section{MATERIAL AND METHODS}

The branches and mature fruits were collected from five trees of Boswellia serrata Roxb of compartment PF318, Kawardha forest division, Chhattisgarh, India (GPS coordinate: N22. 09803 E081.17348) in the month of April ( $1^{\text {st }}$ week). From each tree, five cuttings of $25 \mathrm{~cm}$ length and different thickness $(1-2 \mathrm{~cm}$ diameter) were prepared and after surface sterilization with fungicide (Bavistine) those were treated with Indole-3-Acetic Acid (IAA) and Indole-3-Butyric Acid (IBA) of 500 ppm, 1000 ppm, 2000 ppm and 4000 ppm concentration respectively (Fig. 1H). The eight bunches of cuttings were kept overnight treated with growth hormones IAA and IBA and planted in root trainers filled with the potting mixture soil, vermicompost, and sand (1:1:2). On the other hand, one huge branch cutting from each tree with $1 \mathrm{~m}$ of length and $4 \mathrm{~cm}$ of basal diameter were also prepared for planting (Fig. 1B,C). These cuttings were planted within same potting mixture given above, planting $25 \mathrm{~cm}$ deep without application of any growth regulator. Five tri-lobbed fruits from each tree were depulped and kernels were sown in different directions (25 seeds upward and 25 seeds downward) within the same potting mixture. The planted materials were kept in the shade house and watered daily. The regeneration and growth data were recorded daily.

\section{RESULT AND DISCUSSION}

After 50 days of planting, no growth or differentiation could be observed in $25 \mathrm{~cm}$ long sized cuttings. All seeds germinated but could grow not more than the state of small plantlets. Three out of five $1 \mathrm{~m}$ length branch cuttings of thick diameter survived and first bud was initiated after 12 days of planting. First, leaf differentiation 
was observed after 22 days, shoot proliferation was observed after 47 days (Fig. 1D-E). After 65 days of planting the branch, cuttings were dug out and established rooting was confirmed (Fig. 1F).

The present investigation briefs the importance of larger explants size for rooting in Boswellia serrata Roxb. The small-sized branch cuttings contain high quantity of gum-resin that might check the rooting even after treatment of plant growth regulators or by another mean. Khan (1972) endorsed the selection of 100-125 cm long and 20-25 cm thick branch cuttings for clonal regeneration of Boswellia papyrifera but the same could not be replicated for Boswellia serrata due to the high content of gum-resin oozes out from the sap.

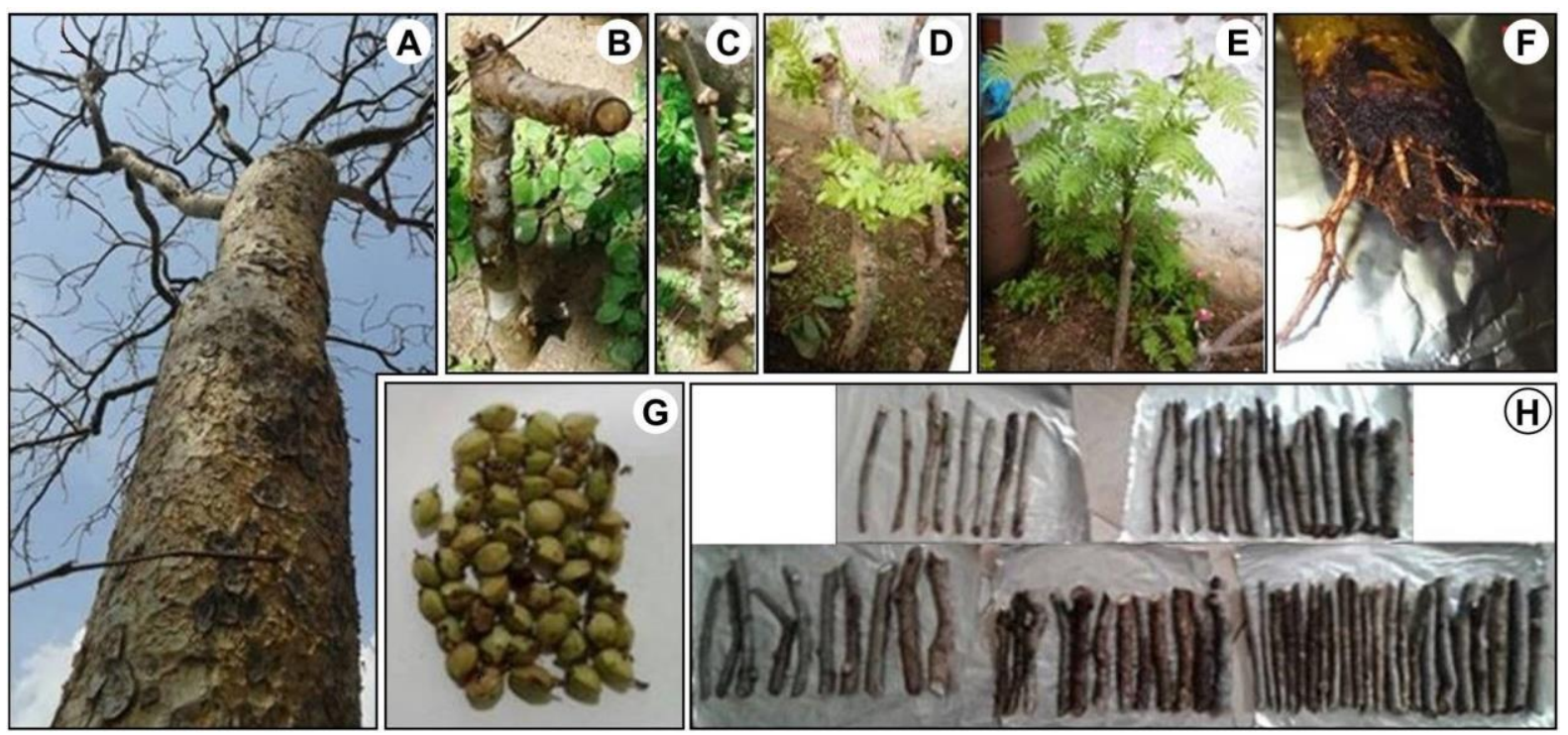

Figure 1. Boswellia serrata Roxb.: A, A view of a tree trunk; B-C, thick and long branch cuttings; D-E, Shoot proliferation from large sized cuttings; F, Root proliferation from large size cuttings after 65 days; G, Seeds used for planting; H, $25 \mathrm{~cm}$ long cuttings.

\section{CONCLUSION}

Although, success achieved with small number of cuttings, the experiment needs to perform further. The successful rooting from the large size (thicker $>4 \mathrm{~cm}$ and long $>1 \mathrm{~m}$ ) branch cuttings can be investigated in an extended manner.

\section{ACKNOWLEDGEMENT}

The fund support from the SFRTI, Chhattisgarh is gratefully acknowledged.

\section{REFERENCES}

Ammon HP (2006) Boswellic acid in chronic inflammatory disease. Plantation Medicines 72(12): 1100-1116.

Ghorpade RP, Chopra A \& Nikam TD (2010) In vitro zygotic embryo germination and propagation of an endangered Boswellia serrata Roxb., a source of boswellic acid. Physiology and Molecular Biotechnology of Plant 16(2): 159-165.

Joshi HB (1981) Troup's Silviculture of Indian trees, Vol. III. The Controller of Publications, New Delhi. Kapoor LD (1990) CRC Handbook of ayurvedic medicinal plants. CRC Press, Baca Raton, Florida.

Khan MAW (1972) Propagation of Boswellia papyrifera through branch-cuttings. Indian Forester 98(7): 437-440.

Purohit SD, Tak K \& Kukad G (1995) In vitro propagation of Boswellia serrata Roxb., Biologia Plantarum 37(3): 35-340.

Saha D, Ved D, Ravikumar K \& Haridasan K (2015) Boswellia ovalifoliolata. The IUCN Red List of Threatened Species 2015: e.T50126567A50131280. Available from: http://dx.doi.org/10.2305/IUCN.UK. 2015- 2.RLTS.T50126567A50131280.en. (accessed: 15 Oct. 2017).

Shah SA, Rathod IS, Suhagia BN, Pandya SS \& Parmar VK (2008) A simple high-performance liquid chromatography for the estimation of boswellic acids from the market formulations containing Boswellia serrata extract. Journal of Chromatography Science 46(8): 735-738.

Sunnichan VG, Mohan Ram HY \& Shivanna KR (2005) Reproductive biology of Boswellia serrata, the source of salai guggul, an important gum-resin. Botanical Journal of the Linnean Society 147(1): 73-82. 